\title{
ISUAL Imager and far-ultraviolet spectrophotometer degradation
}

\author{
Shu-Chun Chang ${ }^{1, *}$, Rue-Ron Hsu ${ }^{1}$, Han-Tzong Su${ }^{1}$, Alfred B. Chen ${ }^{2}$, Cheng-Ling Kuo ${ }^{3}$, \\ Jung-Kung $\mathrm{Chou}^{1}$, Yen-Jung $\mathrm{Wu}^{1}$, and Kang-Ming Peng ${ }^{1}$ \\ ${ }^{1}$ Department of Physics, National Cheng Kung University, Tainan City, Taiwan \\ ${ }^{2}$ Institute of Space and Plasma Sciences, National Cheng Kung University, Tainan City, Taiwan \\ ${ }^{3}$ Institute of Space Science, National Central University, Taoyuan City, Taiwan
}

Article history:

Received 29 February 2016

Revised 19 August 2016

Accepted 26 August 2016

Keywords:

Imager of Sprites and Upper Atmospheric Lightning (ISUAL), Transient luminous events (TLEs), Far-ultraviolet spectrophotometer, Elves

Citation:

Chang, S.-C., R.-R. Hsu, H.-T. Su, A. B. Chen, C.-L. Kuo, J.-K. Chou, Y.-J. Wu, and K.-M. Peng, 2017: ISUAL Imager and far-ultraviolet spectrophotometer degradation. Terr. Atmos. Ocean. Sci., 28, 563-569, doi: 10.3319/TAO.2016.08.26.01

\begin{abstract}
The Imager of Sprites and Upper Atmospheric Lightning (ISUAL) has been operated for more than ten years. The onboard spectrophotometer channel-1 (SP1) was designed to detect the far-ultraviolet (FUV) emissions $\left(\mathrm{N}_{2}\right.$ Lyman-Birge-Hopfield band) from space. It was found that most of the transient luminous events (TLEs), such as sprites, halos, elves, and gigantic jets, are usually accompanied by FUV emissions. The FUV emission could be utilized as an indicator for searching TLEs because the FUV emission of lightning is severely attenuated in the atmosphere during propagation, although the TLE FUV emissions are still detectable. Furthermore, the elves FUV emission photon flux can also be used to estimate the parent lightning peak current. Therefore, SP1 sensitivity degradation assessment, which affects the observed intensity of FUV emissions, is important. Although ISUAL has a lightemitting diode (LED) which is preset as a standard light source, the emission wavelength range of the LED does not cover the FUV band. We established a method in this study to determine the ISUAL Imager and SP1 sensitivity degradation. We found that the annual degradation rates of the ISUAL Imager and the SP1 are 8.4 and 9.7\%, respectively.
\end{abstract}

\section{INTRODUCTION}

Transient terrestrial far-ultraviolet emission observation is one of the main missions of the Imager of Sprites and Upper Atmospheric Lightning (ISUAL) payload on board FORMOSAT-2. The far-ultraviolet (FUV) emissions emitted by transient luminous events (TLEs) originated in the Lyman-Birge-Hopfield (LBH) band of nitrogen molecule $\mathrm{N}_{2}$. From the ISUAL data analyses, it is known that elves (Mende et al. 2005; Kuo et al. 2007, 2008; Chang et al. 2010), halos (Frey et al. 2007; Kuo et al. 2008), sprites (Kuo et al. 2005, 2008; Liu and Pasko 2005; Liu et al. 2006), and gigantic jets (Kuo et al. 2008, 2009; Chou et al. 2010) are accompanied by FUV emissions. However, blue jets without the FUV emission were also reported (Chou et al. 2010) as the longer life time of the $\mathrm{N}_{2} \mathrm{LBH}$ band upper state results in quenching at the lower altitudes.

The ISUAL global observations results show that elves

\footnotetext{
* Corresponding author

E-mail:pollyce@phys.ncku.edu.tw
}

is the most frequently observed occurrence type of TLEs (Chen et al. 2008). Elves is induced by the electromagnetic pulses emitted by an intense lightning (Inan et al. 1991) and was first observed by Fukunishi et al. (1996). Both the theoretical and experimental results show that the time-integrated photon flux of elves in both the $\mathrm{N}_{2}$ first positive (1P) band and $\mathrm{N}_{2}$ Lyman-Birge-Hopfield (LBH) bands depends on the lightning peak current (Barrington-Leigh and Inan 1999; Kuo et al. 2007; Chang et al. 2010; Chen et al. 2014). Owing to the proximity between the elves and the causative lightning, the spectrophotometric observations of the elves $\mathrm{N}_{2} 1 \mathrm{P}$ optical emissions are always contaminated by the lightning emissions except for the elves triggered by lightning located behind the Earth's limb. For the elves triggered by lightning occurring in front of the Earth's limb, the FUV emission could be an indicator of the elves emission intensity because the lightning emission in the FUV band is severely attenuated by the atmospheric molecules (Chang et al. 2010). In addition, from the $\mathrm{N}_{2} \mathrm{LBH}$ intensities of elves 
occurring in the tropical and high latitude regions, Lee et al. (2010) found that the tropical convective system tends to produce brighter elves.

The ISUAL instruments have been operated for over ten years. The absolute sensitivity of each sensor was obtained during the pre-flight lab calibration. The ISUAL instruments were equipped with a light-emitting diode (LED) as a calibration source. The LED degradation is negligible because the total radiation dose endured in space during a 5 -year mission is less than $5 \mathrm{krad}$. This dose is insufficient to cause significant degradation (Chen et al. 2012). Chen et al. (2012) estimated the annual ISUAL sensitivity degradation rate, except for the Imager micro-channel plate (MCP) and the channel-1 of the spectrophotometer (SP1: $150-280 \mathrm{~nm}$ ). The difficulties in estimating the Imager and SP1 degradation rate come from the calibrator low brightness that cannot be detected by the Imager and from the limited LED emission wavelength range that does not cover the FUV band. In many space missions, the known celestial sources, like the Moon, are used for sensor calibrations. The moonlight spectrum is similar to that of the Sun and covers well the SP1 bandwidth without any atmospheric absorption. However, the moonlight is too strong for ISUAL sensors and may cause permanent damage to the electron multiplying devices. Thus, we had to close the sensors when the Moon was in their field of view. A new standard light source is needed for calibrating the Imager and SP1. Due to the wellinvestigated emission intensities of elves (Kuo et al. 2007) and their high occurrence rate (Chen et al. 2008); elves can be used as a calibrator. This paper presents a method to estimate the degradation rate using the elves emissions as a calibrator, and the ISUAL Imager sensitivity degradation as well as the SP1 is also discussed.

\section{INSTRUMENTS AND DATA REDUCTION}

The ISUAL contains three sensors: an intensified CCD imager, a six-channel spectrophotometer (SP) and a dualmodule array photometer (AP) (Chern et al. 2003). The Imager is equipped with a microchannel plate and the SP channels use photomultiplier tubes (PMT) as photon detectors to detect low level light events. The ISUAL has been operated for more than ten years since May 2004. The sensitivity degradation needs to be assessed for the correct imaging and photometric analyses. The ISUAL Imager field of view $(\mathrm{FOV})$ is $20^{\circ}(H) \times 5^{\circ}(\mathrm{V})$. The images used in this study were obtained with a $633-751 \mathrm{~nm}$ filter $\left(\mathrm{N}_{2} 1 \mathrm{P}\right)$ and a frame integration time of $29 \mathrm{~ms}$. Each SP channel has approximately the same FOV as the Imager and records the photometric variations with a $10 \mathrm{kHz}$ sampling rate. Only the SP1 and the SP4 data were used in this study. SP1 is equipped with an FUV filter $(150-290 \mathrm{~nm})$ to detect photons in the $\mathrm{N}_{2}$ LBH band. The SP4 channel has a broadband filter (624 - $750 \mathrm{~nm}$ ) for $\mathrm{N}_{2} 1 \mathrm{P}$ band emission detection. The ISUAL
Imager, SP1 and SP4 bandwidths are shown in Fig. 1.

The sensor band pass and the transmittance along the line of sight affected the emission band contribution percentage to the ISUAL Imager, the SP1, and the SP4. The emission band contribution percentage is defined by

$B_{k}(h, L)=\frac{\sum_{\lambda} I_{k}(\lambda) T(\lambda, h, L) R(\lambda) q_{k}(h) \Delta \lambda}{\sum_{\lambda} I_{k}(\lambda) \Delta \lambda}$

where $I_{k}(\lambda)$ is the band emission from $\mathrm{N}_{2} 1 \mathrm{P}$ or $\mathrm{N}_{2} \mathrm{LBH}$ given in the work by Kuo et al. (2008), and $R(\lambda)$ is the relative sensor response function is shown in Fig. 1. The atmospheric transmittance, $T(\lambda, h, L)$, is a function of the wavelength $\lambda$, the assumed altitude $h$ of elves and the distance $L$ between the elves and the satellite. The transmittance calculation follows Lambert's law and the parameters are the same as that used in a work by Kuo et al. (2008 and the references therein). The quenching factor $q_{k}(h)$ is defined by the same formula and parameters used in a work by Kuo et al. (2008, and the references therein). The band contribution $B_{k}$ for the chosen events that occurred at $90 \mathrm{~km}$ is shown in Fig. 2.

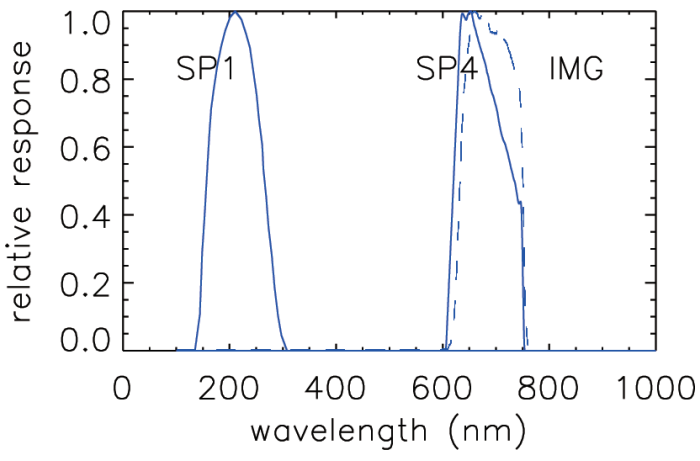

Fig. 1. The relative response curve of the ISUAL imager with filter \#1 (633 - $751 \mathrm{~nm}$ ), the SP channel 1 and 4. (Color online only)

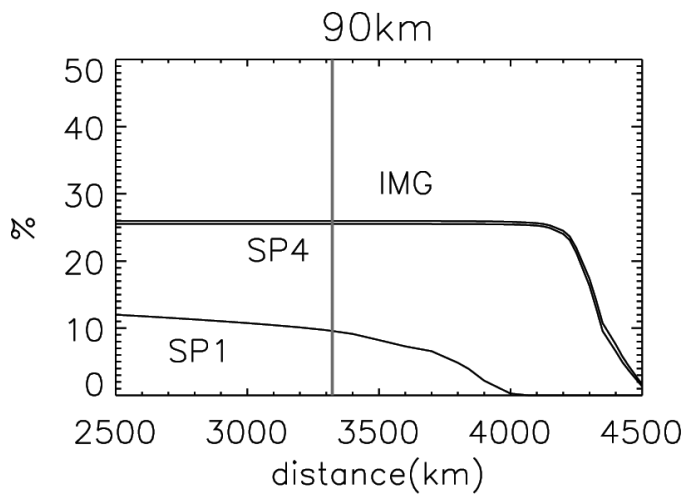

Fig. 2. The percentage contribution of molecular nitrogen bands to the SP channels and the Imager (IMG) in the situation that the event occurred at $90 \mathrm{~km}$ altitude. The gray line shows the boundary between in-front-of-the-limb and behind-the-limb. 
The measured SP amplitude (digital counts) can be converted into the meaningful scientific values (photons $\mathrm{cm}^{-2} \mathrm{sec}^{-1}$ ) using the absolute sensor sensitivity measured during the pre-flight calibration. The SP4 PMT sensitivity degrades by $\sim 7.7 \%$ annually (Chen et al. 2012, Fig. 3 ). The light source used here is elves because the elves optical intensity was well described (Kuo et al. 2007). We estimated the calibrated SP4 photon fluxes for the elves by assuming the negligible optics degradation (lenses and filters) and by considering the PMT sensitivity degradation. In the following subsections, the elves emissions in the Imager, the SP4, and the SP1 are analyzed in detail.

\subsection{The Imager Degradation}

The $\mathrm{N}_{2} 1 \mathrm{P}$ emission band contributions to the ISUAL Imager and to the SP4 are approximately the same, as shown in Fig. 2. The SP4 degradation rate was estimated in the work by Chen et al. (2012). Thus, the elves photon fluxes obtained from the SP4 can be regarded as a standard light source to calibrate the Imager. Note that the ISUAL is configured with the eastward view observing TLEs occurring at a distance of $2300-4400 \mathrm{~km}$. For elves induced by the lightning located in front of the Earth's limb, the recorded SP4 signals consist of emissions from both the elves and the parent lightning. Thus, we selected the elves triggered by the lightning located behind the limb to avoid the lightning contamination. As an example, the image and the SP4 data for the selected elves induced by lightning located behind the Earth's limb are shown in Fig. 3.

Because of the instrument problem, the filter setting of the ISUAL Imager for the regular observations was changed from filter \#1 ( $\mathrm{N}_{2} 1 \mathrm{P}$ band) to filter \#6 (broad band without filter) since 2012. Therefore, we chose the elves data set recorded from the beginning of 2004 until the end of 2011 to study the Imager degradation. ISUAL recorded 15849 elves from 2004 - 2011. Three hundred and eighty-two elves were triggered by lightning located behind the Earth's limb (3700 - $4100 \mathrm{~km}$ away from the ISUAL) and detected well within the FOV of the ISUAL Imager and SP.

The total elves photon flux in the SP4 is obtained by integrating the SP4 waveform. The result was corrected by taking into account the degradation factor determined by Chen et al. (2012, their Fig. 3). The corrected total photon flux in SP4 $\left(\Lambda_{S P 4, s t d}\right)$ could be used as the standard light source. The total elves intensity on the image frame was obtained using the differential photometry method. The procedures are as follows: (1) Removing the background by subtracting the previous frame from the trigger frame. (2) Selecting a region, as shown in Fig. 3, which includes elves but excludes the lightning in the trigger frame. (3) Calculating the background noise standard deviation $\left(\sigma_{I M G}\right)$ and selecting the pixels with intensity larger than $\sigma_{I M G}$. (4) Converting counts into photons $\mathrm{cm}^{-2} \mathrm{~s}^{-1} \mathrm{sr}^{-1}$ by applying the sensitivity
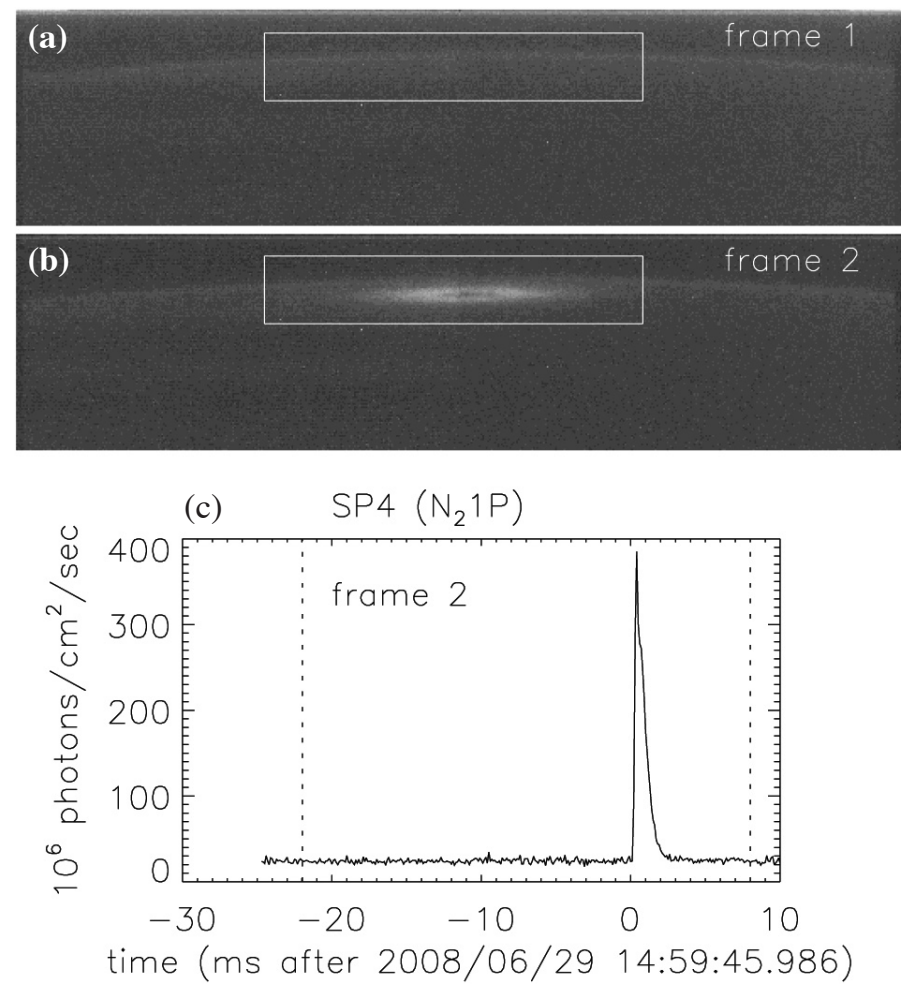

Fig. 3. Elves triggered by lightning located behind the Earth's limb, occurring on 2008/06/29 14:59:45.986 UTC. (a) The pre-trigger frame, (b) the trigger frame, and (c) the associated $\mathrm{N}_{2} 1 \mathrm{P}$ signal of the SP4 signal around the trigger time. 
curve obtained in the pre-flight calibration. The luminosity of each selected pixel is represented by $I_{(i, j)}$, where $(i, j)$ represents the coordinates of the selected pixel. (5) The total elves photon flux was obtained by integrating $I_{(i, j)}$ spatially and temporally, that is,

$\Lambda_{I M G}=\sum_{(i, j)} I_{(i, j)} \Omega_{p i x e l} \Delta t$

where $\Omega_{\text {pixel }}$ is the solid angle of one pixel, and $\Delta t$ is the integrating time of one frame. The photon flux error is given by

$\sigma_{\Lambda_{M M G}}=\sigma_{I M G} \times \sum_{(i, j)} \Omega_{p i x e l} \Delta t$

The image photon flux ratio to the corrected SP4 photon flux of the elves $\left(\Lambda_{I M G} / \Lambda_{S P 4, s t d}\right)$ at different mission elapsed time (MET) are shown in Fig. 4a. The MET is counted by the day after the FORMOSAT-2 launched on 21 May 2004. By considering the bandpass, the $\mathrm{N}_{2} 1 \mathrm{P}$ emissions recorded by the SP4 and by the Imager would be approximately identical. However, as shown in Fig. 4a, the elves photon flux on the image frame is larger than that in the SP4. This discrepancy may come from the temperature changes between calibration and on orbit operations. This was mentioned by Mende et al. (2005). Using the error propagation equation, the error bar of each data points is calculated using the following formula:

$\sigma_{\Lambda_{M M G} / \Lambda_{S P 4, s d}}=\frac{\Lambda_{I M G}}{\Lambda_{S P 4, s t d}}\left[\left(\frac{\sigma_{\Lambda_{M M G}}}{\Lambda_{I M G}}\right)^{2}+\left(\frac{\sigma_{\Lambda_{S P A, s t}}}{\Lambda_{S P 4, s t d}}\right)^{2}\right]^{1 / 2}$

where $\sigma_{\Lambda_{\text {MG }}}$ and $\sigma_{\Lambda_{\text {SPA } \text { Ud }}}$ denote the photon flux errors measured in the Imager and the SP4, respectively.

If the Imager sensitivity is steady, the photon flux ratio $\Lambda_{I M G} / \Lambda_{S P 4, \text { std }}$ should also remain constant. However, as seen in Fig. 4a, the ratio $\Lambda_{I M G} / \Lambda_{S P 4, \text { std }}$ decreases with the MET.
The Imager sensitivity degradation rate can be inferred using the $\Lambda_{I M G} / \Lambda_{S P 4, \text { std }}$ decreasing ratio. We followed the fitting formula established by Chen et al. (2012) to quantify the Imager degradation rate. The sensor's efficiency decays exponentially with time and is expressed in the form of

$\mathrm{r} \propto e^{-\alpha t}$

where $t$ is the MET in units of days, and $\alpha$ is the fitting coefficient. The $\Lambda_{I M G} / \Lambda_{S P 4, \text { std }}$ ratio is found to decrease with the rate of $\alpha=0.00024$. The fitting curve is normalized to the remaining efficiency defined as $100 \%$ at $\mathrm{MET}=0$. The remaining Imager efficiency is displayed in Fig. 4b. It shows that the annual Imager sensitivity compound degradation rate is estimated to be $\sim 8.4 \%$.

The annual Imager degradation rate includes the MCP and optics degradation. The degradation of the SP4 optics was neglected in our estimation of $\Lambda_{S P 4, \text { std }}$. If the SP4 optics degradation was not neglected, both $\Lambda_{S P 4, \text { std }}$ and the Imager degradation rate would be underestimated.

\subsection{The Spectrophotometer Channel 1 Degradation}

The channel 2 to 6 calibration for the ISUAL SP was analyzed by Chen et al. (2012). The calibrations were run through measuring the built-in quick-blinking LED calibrators (Chen et al. 2012). However, the LED calibrator emissions were blocked by the FUV filter for the SP1. Another light source, i.e., elves, was selected as the calibrator because of its well-investigated emission properties. Note that the SP1 band contribution significantly decreases with increasing distance. If the event is triggered by the lightning located behind the limb, it will endure severe atmospheric absorption (Fig. 2). As the average geolocation error, which is about $50 \mathrm{~km}$ pixel $^{-1}$ along the line of sight (Chen et al. 2008), it will also induce a larger uncertainty for estimating
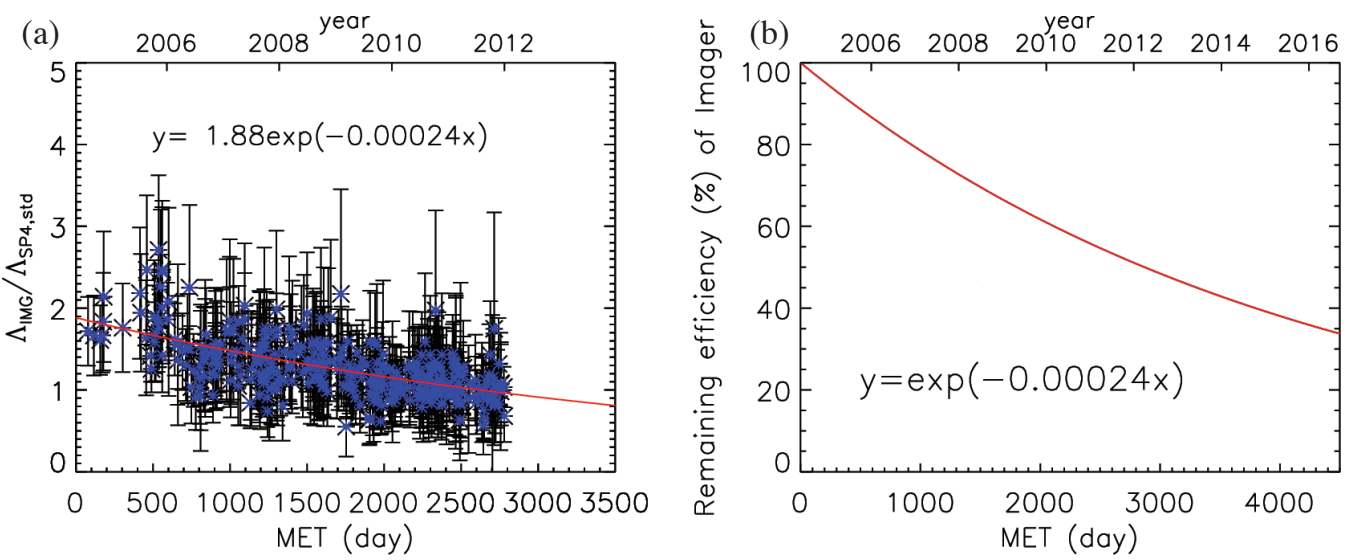

Fig. 4. (a) The time variation for the ratio between the $\mathrm{N}_{2} 1 \mathrm{P}$ photon flux of the Imager and the calibrated $\mathrm{N}_{2} 1 \mathrm{P}$ photon flux of the SP4 for the elves (b) Remaining efficiency (\%) of the Imager. (Color online only) 
the photon flux in SP1 $\left(\Lambda_{S P 1}\right)$ of the behind-the-limb elves. Therefore, we had to choose elves triggered by lightning located in front of the Earth's limb (2700 - 3000 km away from FORMOSAT-2) for analysis. Under this selection condition, 589 events occurred in the data set. The Imager and SP1 data for the selected elves induced by lightning located in front of the Earth's limb are shown in Fig. 5. Although the parent lightning is also in the FOV of the SP1, the lightning emission in the FUV band is difficult to transmit through the atmosphere from $10 \mathrm{~km}$ altitude to FORMOSAT-2 ( 890 km) (Chang et al. 2010). According to the elves simulation presented by Kuo et al. (2007), a relationship exists between the time-integrated $\mathrm{N}_{2} 1 \mathrm{P}$ photon flux and the time-integrated SP1 FUV photon flux. Hence, we can estimate the standard total photon flux in SP1.

We followed the procedures presented in the previous subsection to estimate the Imager $\mathrm{N}_{2} 1 \mathrm{P}$ photon flux $\left(\Lambda_{I M G}\right)$ and the FUV photon flux of the SP1 $\left(\Lambda_{S P 1}\right)$ of the selected elves. The photon flux of the elves in the Imager was corrected as the standard light source $\left(\Lambda_{I M G, \text { std }}\right)$ by applying the Imager sensitivity degradation rate determined in the subsection 2.1. The uncertainty in the elves standard photon flux estimation in the Imager $\left(\sigma_{\Lambda_{I M G, s d}}\right)$ was obtained by dividing $\sigma_{\Lambda_{I M G}}$ by the degradation factor. Based on the elves simulation model presented by Kuo et al. (2007), the theoretical relationship between the $\mathrm{N}_{2} 1 \mathrm{P}$ photon flux in the SP4 $\left(\Lambda_{S P 4}\right)$ and the FUV photon flux in the SP1 $\left(\Lambda_{S P 1}\right)$ could be obtained and is shown in Chang et al. (2010, dashed line in their Fig. 4a). According to the result in subsection 2.1, the $\mathrm{N}_{2} 1 \mathrm{P}$ photon flux in the Imager is $\sim 1.88$ times the $\mathrm{N}_{2} 1 \mathrm{P}$ photon flux in the SP4. By taking into account this factor, the standard photon flux of the elves in the Imager could be converted into the standard photon flux of the elves in SP1 $\left(\Lambda_{S P 1, s t d}\right)$. The error in the estimated standard photon flux for the elves $\left(\sigma_{\Lambda_{S P 1, \text { ssd }}}\right)$ can be estimated using $\sigma_{\Lambda_{M G \text {, std }}}$ and the error propagation method.

The ratio of $\Lambda_{S P 1} / \Lambda_{S P 1, \text { std }}$ is shown in Fig. 6a. The error bars in the ratio are obtained by considering the propagation error following Eq. (4). As shown in Fig. 6a, the ratio of the SP1 photon flux to the standard SP1 photon flux of elves $\Lambda_{S P 1} / \Lambda_{S P 1, \text { std }}$ decreases monotonically with MET. The decreasing ratio results from the SP1 sensor sensitivity degradation. By fitting the data with the exponential decaying function to estimate the decreasing rate, it is found that the sensitivity decreases with the rate of $\alpha=0.00028$. As the fitting curve is normalized to the remaining efficiency which is defined as $100 \%$ at MET $=0$, the remaining SP1 efficiency is displayed in Fig. 6b. It shows that the compound annual SP1 sensitivity degradation rate is estimated at $9.7 \%$ and is comparable to other SP channels, as shown in Fig. 3 in Chen et al. (2012).

The estimated annual SP1 degradation rate the SP1 PMT and the SP1 optics degradation rates. In our calculation, the SP1 degradation rate was obtained by analyzing the
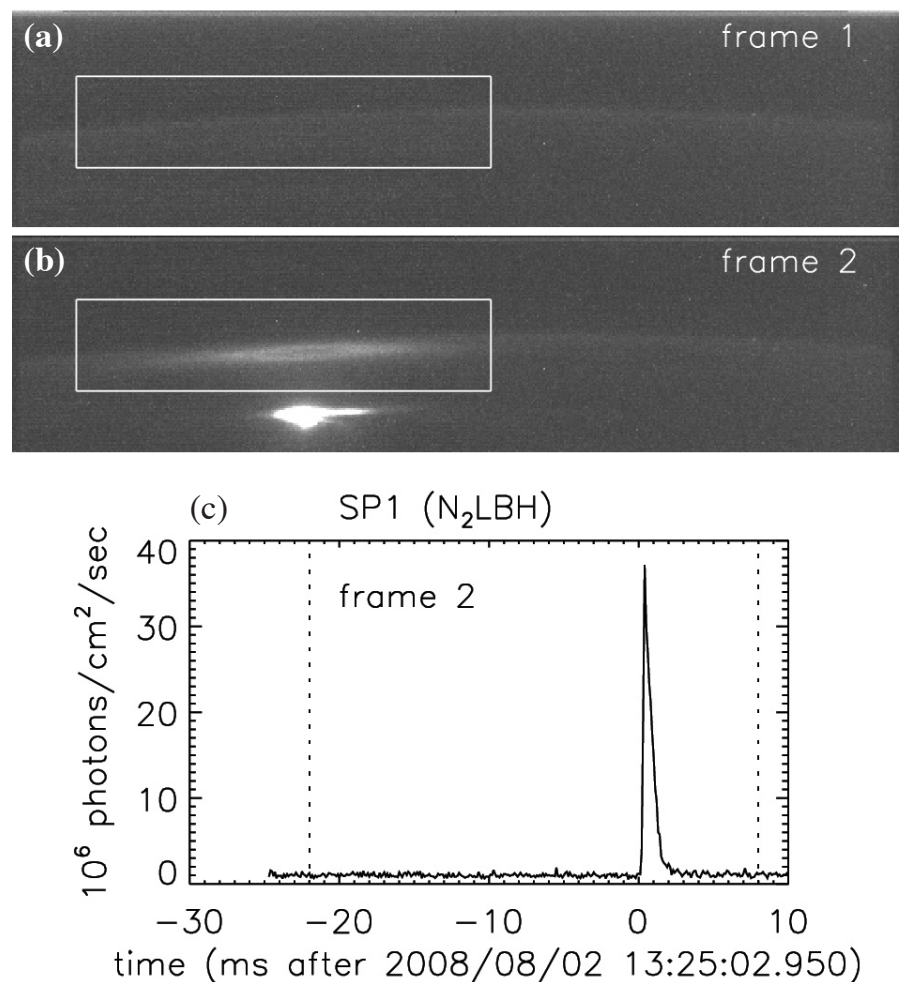

Fig. 5. Elves triggered by lightning located in front of the Earth's limb. (a) The pre-trigger frame, (b) the trigger frame, and (c) the associated FUV signal of the SP1 around the trigger time. 

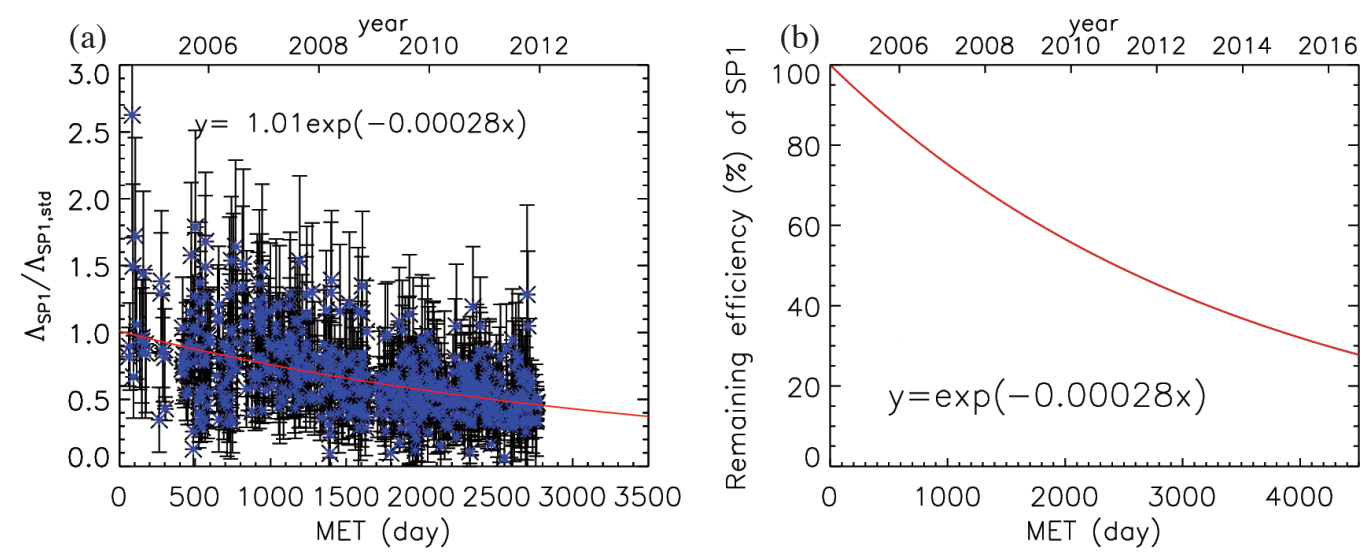

Fig. 6. (a) The time variation for the ratio of the measured to the standard FUV photon flux of the SP1. (b) The remaining efficiency (\%) of the SP1. (Color online only)

ratio of the measured $\Lambda_{S P 1}$ to the estimated $\Lambda_{S P 1, \text { std }}$, where $\Lambda_{S P 1, \text { std }}$ was estimated from the $\Lambda_{I M G, \text { std }}$ value. As we mentioned at the end of Section 2.1, the Imager degradation rate would be underestimated if the SP4 optics degradation could not be neglected. This effect may cause the value of $\Lambda_{I M G \text {,std }}$ as well as $\Lambda_{S P 1, \text { std }}$ and the SP1 degradation rate given in our study to be underestimated. Our estimation could give the least ISUAL SP1 sensor degradation rate for reference.

\section{DISCUSSION AND SUMMARY}

We introduced an indirect but effective method to estimate the ISUAL Imager with filter \#1 (633 - $751 \mathrm{~nm})$ and SP1 $(150-290 \mathrm{~nm})$ degradation rates. Elves were selected as the calibrator because of their well-investigated emission properties. We chose the behind-the-limb elves first, and calculated the Imager $\mathrm{N}_{2} 1 \mathrm{P}$ photon flux as well as the calibrated SP4 $\mathrm{N}_{2} 1 \mathrm{P}$ photon flux for selected elves. The decreasing Imager- $\mathrm{N}_{2} 1 \mathrm{P}$ photon flux ratio to the calibrated SP4- $\mathrm{N}_{2} 1 \mathrm{P}$ photon flux shows that the Imager sensitivity degrades annually with the compound rate of $\sim 8.4 \%$. We next estimated the $\mathrm{N}_{2} \mathrm{LBH}$ photon flux of the SP1 and the standard SP1 photon flux to determine the SP1 sensitivity degradation rate. The compound annual SP1 degradation rate is about $9.7 \%$. The sensitivity degradation rates of the ISUAL Imager and the SP1 are comparable to other SP channels reported by Chen et al. (2012). From the annual Imager and SP1 degradations, the ISUAL Imager and the SP1 sensitivity will reduce to less than 70 and $60 \%$ after 5 years operation, respectively. This fact means that the Imager and SP1 degradations should be seriously considered in the FORMOSAT-2 ISUAL data analyses.

The SP1 degradation rate estimation is useful for the ISUAL and also for other space missions. For instance, another space missions with TLE research, JEM-GLIMS (Global Lightning and Sprite Measurements of Japanese Experiment Module), was also equipped with the same FUV detecting sensor (Sato et al. 2015). As the JEM-GLIMS was nadir viewing rather than limb viewing like the ISUAL, the method for estimating the SP1 degradation rate presented in this paper would be difficult to carry out. However, the ISUAL SP1 degradation rate estimated in this paper would be an important reference for considering the JEM-GLIMS FUV sensor degradation rate.

Acknowledgements These works were supported in part by the National Space Organization (NSPO) and National Science Council (NSC) in Taiwan under grants MOST 105-2811-M-006-053, MOST 105-2811-M-006-007, NSPO-S-104013, MOST 104-2111-M-006-004, MOST 104-2111-M-006-005-MY2, MOST 104-2119-M-006-020, MOST 102-2112-M-006-017-MY3.

\section{REFERENCES}

Barrington-Leigh, C.P. and U. S. Inan, 1999: Elves triggered by positive and negative lightning discharges. Geophys. Res. Lett., 26, 683-686, doi: 10.1029/1999GL900059. [Link]

Chang, S. C., C. L. Kuo, L. J. Lee, A. B. Chen, H. T. Su, R. R. Hsu, H. U. Frey, S. B. Mende, Y. Takahashi, and L. C. Lee, 2010: ISUAL far-ultraviolet events, elves, and lightning current. J. Geophys. Res., 115, A00E46, doi: 10.1029/2009ja014861. [Link]

Chen, A. B., C. L. Kuo, Y. J. Lee, H. T. Su, R. R. Hsu, J. L. Chern, H. U. Frey, S. B. Mende, Y. Takahashi, H. Fukunishi, Y. S. Chang, T. Y. Liu, and L. C. Lee, 2008: Global distributions and occurrence rates of transient luminous events. J. Geophys. Res., 113, A08306, doi: 10.1029/2008JA013101. [Link]

Chen, A. B. C., Y. Y. Wu, C. Y. Chiang, Y. C. Huang, C. C. Kuo, H. T. Su, R. R. Hsu, S. B. Mende, H. U. Frey, S. E. Harris, Y. Takahashi, and L. C. Lee, 2012: Sensitivity degradation of ISUAL instruments and its impact 
on observations. Terr. Atmos. Ocean. Sci., 23, 71-83, doi: 10.3319/TAO.2011.06.20.01(AA). [Link]

Chen, A. B. C., H. T. Su, and R. R. Hsu, 2014: Energetics and geographic distribution of elve-producing discharges. J. Geophys. Res., 119, 1381-1391, doi: 10.1002/2013JA019470. [Link]

Chern, J. L., R. R. Hsu, H. T. Su, S. B. Mende, H. Fukunishi, Y. Takahashi, and L. C. Lee, 2003: Global survey of upper atmospheric transient luminous events on the ROCSAT-2 satellite. J. Atmos. Sol.-Terr. Phys., 65, 647-659, doi: 10.1016/S1364-6826(02)00317-6. [Link]

Chou, J. K., C. L. Kuo, L. Y. Tsai, A. B. Chen, H. T. Su, R. R. Hsu, S. A. Cummer, J. Li, H. U. Frey, S. B. Mende, Y. Takahashi, and L. C. Lee, 2010: Gigantic jets with negative and positive polarity streamers. J. Geophys. Res., 115, A00E45, doi: 10.1029/2009JA014831. [Link]

Frey, H. U., S. B. Mende, S. A. Cummer, J. Li, T. Adachi, H. Fukunishi, Y. Takahashi, A. B. Chen, R. R. Hsu, H. T. Su, and Y. S. Chang, 2007: Halos generated by negative cloud-to-ground lightning. Geophys. Res. Lett., 34, L18801, doi: 10.1029/2007GL030908. [Link]

Fukunishi, H., Y. Takahashi, M. Kubota, K. Sakanoi, U. S. Inan, and W. A. Lyons, 1996: Elves: Lightninginduced transient luminous events in the lower ionosphere. Geophys. Res. Lett., 23, 2157-2160, doi: 10.1029/96GL01979. [Link]

Inan,U.S., T.F. Bell, and J.V.Rodriguez, 1991: Heating and ionization of the lower ionosphere by lightning. Geophys. Res.Lett., 18, 705-708, doi: 10.1029/91GL00364. [Link]

Kuo, C. L., R. R. Hsu, A. B. Chen, H. T. Su, L. C. Lee, S. B. Mende, H. U. Frey, H. Fukunishi, and Y. Takahashi, 2005: Electric fields and electron energies inferred from the ISUAL recorded sprites. Geophys. Res. Lett., 32, doi: 10.1029/2005g1023389. [Link]

Kuo, C. L., A. B. Chen, Y. J. Lee, L. Y. Tsai, R. K. Chou, R. R. Hsu, H. T. Su, L. C. Lee, S. A. Cummer, H. U. Frey, S. B. Mende, Y. Takahashi, and H. Fukunishi, 2007: Modeling elves observed by FORMOSAT-2 satellite. J. Geophys. Res., 112, A11312, doi: 10.1029/2007JA012407. [Link]
Kuo, C. L., A. B. Chen, J. K. Chou, L. Y. Tsai, R. R. Hsu, H. T. Su, H. U. Frey, S. B. Mende, Y. Takahashi, and L. C. Lee, 2008: Radiative emission and energy deposition in transient luminous events. J. Phys. D: Appl. Phys., 41, 234014, doi: 10.1088/0022-3727/41/23/234014. [Link]

Kuo, C. L., J. K. Chou, L. Y. Tsai, A. B. Chen, H. T. Su, R. R. Hsu, S. A. Cummer, H. U. Frey, S. B. Mende, Y. Takahashi, and L. C. Lee, 2009: Discharge processes, electric field, and electron energy in ISUAL-recorded gigantic jets. J. Geophys. Res., 114, A04314, doi: 10.1029/2008JA013791. [Link]

Lee, L. J., A. B. Chen, S. C. Chang, C. L. Kuo, H. T. Su, R. R. Hsu, C. C. Wu, P. H. Lin, H. U. Frey, and S. B. Mende, 2010: Controlling synoptic-scale factors for the distribution of transient luminous events. J. Geophys. Res., 115, A00E54, doi: 10.1029/2009JA014823. [Link]

Liu, N. and V.P. Pasko, 2005: Molecular nitrogen LBH band system far-UV emissions of sprite streamers. Geophys. Res. Lett., 32, L05104, doi: 10.1029/2004GL022001. [Link]

Liu, N., V. P. Pasko, D. H. Burkhardt, H. U. Frey, S. B. Mende, H. T. Su, A. B. Chen, R. R. Hsu, L. C. Lee, H. Fukunishi, and Y. Takahashi, 2006: Comparison of results from sprite streamer modeling with spectrophotometric measurements by ISUAL instrument on FORMOSAT-2 satellite. Geophys. Res. Lett., 33, L01101, doi: 10.1029/2005GL024243. [Link]

Mende, S. B., H. U. Frey, R. R. Hsu, H. T. Su, A. B. Chen, L. C. Lee, D. D. Sentman, Y. Takahashi, and H. Fukunishi, 2005: D region ionization by lightning-induced electromagnetic pulses. J. Geophys. Res., 110, A11312, doi: 10.1029/2005JA011064. [Link]

Sato, M., T. Ushio, T. Morimoto, M. Kikuchi, H. Kikuchi, T. Adachi, M. Suzuki, A. Yamazaki, Y. Takahashi, U. Inan, I. Linscott, R. Ishida, Y. Sakamoto, K. Yoshida, Y. Hobara, T. Sano, T. Abe, M. Nakamura, H. Oda, and Z. I. Kawasaki, 2015: Overview and early results of the Global Lightning and Sprite Measurements mission. J. Geophys. Res., 120, 3822-3851, doi: 10.1002/2014JD022428. [Link] 